\title{
Facile synthesis and anticancer activity of novel dihydropyrimidinone derivatives
}

\author{
Mashooq A. Bhat ${ }^{1, *}$, Mohamed A. Al-Omar', Ahmed M. Naglah', Abdullah Al-Dhfyan² \\ ${ }^{1}$ Department of Pharmaceutical Chemistry, College of Pharmacy, King Saud University, Riyadh 11451, Saudi Arabia \\ ${ }^{2}$ Department of Pharmacology and Toxicology, College of Pharmacy, King Saud University, Riyadh 11451, Saudi Arabia \\ "Corresponding author: e-mail: mabhat@ksu.edu.sa
}

\begin{abstract}
The enaminone, $(2 E)-3$-(dimethylamino)-1-(3,4,5-trimethoxyphenyl) prop-2-en-1-one was prepared by refluxing 3,4,5-trimethoxy acetophenone with dimethylformamide dimethylacetal (DMF-DMA) without solvent for $12 \mathrm{~h}$. The dihydropyrimidinone derivatives (1-9) were prepared by reacting enaminone, substituted benzaldehydes and urea in glacial acetic acid. The compounds (1-9) were synthesized in significant yield using one step multicomponent reaction. Structures of all the novel synthesized compounds were characterized and confirmed by various spectroscopic methods. The compounds were evaluated for their anti-cancer activity against HepG2 cancer cell line. Compound 9 displayed significant anti-cancer activity. During the apoptotic assay, it showed a significant increase in necrosis from $1.97 \%$ to $12.18 \%$ as compared to the control. Mechanism of anti-proliferation was performed by cell cycle distribution assay, which showed a decrease in $\mathrm{G} 2+\mathrm{M}$ from 12.90 to 8.13 as compared to control.
\end{abstract}

Keywords: Dihydropyrimidinone; enaminone; 3,4,5-trimethoxybenzoyl moiety.

\section{INTRODUCTION}

Pyrimidines have played a significant role in medicinal chemistry ${ }^{1}$. Pyrimidines are important scaffolds because of their antihypertensive activity ${ }^{2}$. In 1975, nifedipine (4-aryl-1,4-dihydropyridine) was first introduced as an antihypertensive drug into clinical medicine. The dihydropyridines are reported as the most potent calcium channel modulators ${ }^{3}$. Dihydropyrimidinones have been reported as antihypertensive agents ${ }^{4-5}$. The Biginelli reaction involves one-pot synthesis of 3,4-dihydropyrimidine-2 $(1 H)$-ones using aldehydes, active methylene compounds and (thio) urea in an acidic environment. Dihydropyrimidines are associated with a wide spectrum of pharmacological activities ${ }^{6-7}$. In drug development, dihydropyrimidine derivatives have been tested for antibacterial ${ }^{8-9}$, anti-inflammatory ${ }^{10}$, anticancer ${ }^{11}$, anti-parkinson ${ }^{12}$, antidiabetic ${ }^{13}$, antihypertensive ${ }^{14}$ and antitumor activities ${ }^{15}$. The dihydropyrimidinone derivatives have been reported as potent compounds against HepG2 cancer cell line ${ }^{\mathbf{1 6}}$.

3,4,5-Trimethoxy benzoyl moiety has also played a significant role in medicinal chemistry and has been found in several compounds having anti-cancer activity ${ }^{17-18}$. The compounds containing these two important scaffolds (dihydropyrimidinone and 3,4,5-trimethoxybenzoyl) may have significant therapeutic potential as anticancer agents.

In the present study, a novel series of dihydropyrimidinone derivatives $(1-9)$ were synthesized from enaminone, (2E)-3-(dimethylamino)-1-(3,4,5-trimethoxyphenyl) prop-2-en-1-one, analyzed by various spectral techniques and screened for anti-cancer activity against HepG2 cancer cell line ${ }^{19-23}$.

\section{EXPERIMENTAL}

\section{Chemistry}

All the solvents were obtained from (Merck, New Jersy USA). Thin-layer chromatography (TLC) was performed on Silica gel $60 \mathrm{~F}_{254}$ (Merck, Millipore, Billerica, MA, USA). Perkin Elmer FT-IR spectrophotometer (Per-
kinElmer Inc., Walthum, Ma, USA) was used for IR spectroscopy. Gallenkamp melting point apparatus was used for melting point determination. ${ }^{1} \mathrm{H}$ and ${ }^{13} \mathrm{C}$ NMR spectra of the compounds were performed on Bruker NMR 500/700 MHz (Bruker Corporation, Billericia, MA, USA). Mass spectra of compounds were performed on Agilent triple quadrupole $6410 \mathrm{TQ}$ GC/MS equipped with ESI (electrospray ionization). The elemental analysis of the compounds was performed by CHN Elementar (Analysensysteme GmbH, Langenselbold, Germany).

\section{Synthetic procedure}

Synthesis of the (2E)-3-(dimethylamino)-1-(3,4,5-trimethoxyphenyl) prop-2-en-1-one (II)

A mixture of 3,4,5-trimethoxy acetophenone (I) $(0.02$ mol) and dimethylformamide-dimethylacetal (DMF-DMA) $(0.023 \mathrm{~mol})$ was refluxed for $12 \mathrm{~h}$ without solvent. The precipitate was obtained by adding diethyl ether to the reaction mixture. Vacuum filtration was performed to obtain the dry product. The obtained product was recrystallized from absolute ethanol. Yield: $90 \%$; M.p.: 128-130 ${ }^{\circ} \mathrm{C}$; IR $(\mathrm{KBr}) \mathrm{cm}^{-1}: 1638(\mathrm{C}=\mathrm{O}), 1543(\mathrm{C}=\mathrm{C})$, $1118(\mathrm{C}-\mathrm{O}) ;{ }^{1} \mathrm{H}$ NMR $\left(\mathrm{DMSO}_{\mathrm{6}}\right) \delta \mathrm{ppm}: 7.71(1 \mathrm{H}, \mathrm{d}$, $J=12 \mathrm{~Hz},-\mathrm{CH}), 7.1(2 \mathrm{H}, \mathrm{m}, \mathrm{Ar}-\mathrm{H}), 5.8(1 \mathrm{H}, \mathrm{d}, J=12$ $\mathrm{Hz},-\mathrm{CH}), 3.8\left(6 \mathrm{H}, \mathrm{s}, 2 \times \mathrm{OCH}_{3}\right), 3.7\left(3 \mathrm{H}, \mathrm{s}, \mathrm{OCH}_{3}\right)$, $3.1\left(3 \mathrm{H}, \mathrm{s}, \mathrm{N}-\mathrm{CH}_{3}\right), 2.9\left(3 \mathrm{H}, \mathrm{s}, \mathrm{N}-\mathrm{CH}_{3}\right) ;{ }^{13} \mathrm{C}$ NMR $\left(\right.$ DMSO- $\left.\mathrm{d}_{6}\right) \delta$ ppm: $184.4(\mathrm{C}=\mathrm{O}), 154.1(\mathrm{Ar}-\mathrm{C}), 152.4$ $(\mathrm{Ar}-\mathrm{C}), 139.8(\mathrm{Ar}-\mathrm{CH}), 135.8(\mathrm{Ar}-\mathrm{CH}), 104.6(\mathrm{C}=\mathrm{C})$, $90.7(\mathrm{C}=\mathrm{C}), 60.0(\mathrm{OCH} 3), 55.9(\mathrm{OCH} 3), 44.4(\mathrm{~N}-\mathrm{CH} 3)$, 37.1 (N-CH3); MS (ESI) m/z: 265.0 [M]+; Analysis: for $\mathrm{C}_{14} \mathrm{H}_{19} \mathrm{NO}_{4}$, calcd. C 63.38, H 7.22, N 5.28\%; found C $63.14, \mathrm{H} 7.20, \mathrm{~N} 5.30 \%{ }^{24}$.

\section{Synthesis of the dihydropyrimidinone derivatives (1-9)}

A mixture of enaminone, (2E)-3-(dimethylamino)1-(3,4,5-trimethoxyphenyl) prop-2-en-1-one (II) (0.01 $\mathrm{mol})$, substituted benzaldehyde $(0.01 \mathrm{~mol})$, urea $(0.01$ $\mathrm{mol})$ and glacial acetic acid $(10 \mathrm{~mL})$, was refluxed for $3 \mathrm{~h}$. The reaction mixture was precipitated by adding ice-cold water. The product was obtained by vacuum 
filtration and washed several times with cold water. The obtained products were recrystallized from ethanol.

4-(2,3,4-Trimethoxyphenyl-5-(3,4,5-trimethoxybenzoyl)-3,4-dihydropyrimidin-2(1H)-one (1)

Yield: $70 \%$; M.p.: $210-212{ }^{\circ} \mathrm{C}$; IR $(\mathrm{KBr}) \mathrm{cm}^{-1}: 3412$ (NH str.), $1700(\mathrm{C}=\mathrm{O}), 1654(\mathrm{C}=\mathrm{O}), 1617(\mathrm{C}=\mathrm{C}), 1126$ (C-O); ${ }^{1} \mathrm{H}$ NMR $\left(500 \mathrm{MHz}, \mathrm{DMSO}-d_{6}\right): \delta=3.77(18 \mathrm{H}$, s, $\left.6 \times-\mathrm{OCH}_{3}\right), 5.57(1 \mathrm{H}, \mathrm{d}, J=2.0 \mathrm{~Hz}, \mathrm{H}-4) ; 6.75-7.15$ $(5 \mathrm{H}, \mathrm{m}, \mathrm{Ar}-\mathrm{H}), 7.50(1 \mathrm{H}, \mathrm{s},=\mathrm{CH}), 9.17(1 \mathrm{H}, \mathrm{bs}, \mathrm{NH}$, $\mathrm{D}_{2} \mathrm{O}$ exchg.), 10.20 (1H, bs, $\mathrm{NH}, \mathrm{D}_{2} \mathrm{O}$ exchg.); ${ }^{13} \mathrm{C} \mathrm{NMR}$ $\left(125.76 \mathrm{MHz}, \mathrm{DMSO}-d_{6}\right): \delta=49.9,56.3,56.5,60.5$, 60.6, 61.2, 106.2, 108.1, 123.0, 129.8, 134.5, 140.2, 142.0, 151.5, 151.5, 153.0, 153.4, 190.9; MS: $\mathrm{m} / \mathrm{z}=458.45$ $[\mathrm{M}]^{+}$; Analysis: for $\mathrm{C}_{23} \mathrm{H}_{26} \mathrm{~N}_{2} \mathrm{O}_{8}$, calcd. C 60.26, H 5.72, N 6.11\%; found C 60.45, H 5.73, N 6.12\%.

4-(2,3-Dimethoxyphenyl-5-(3,4,5-trimethoxybenzoyl)-3,4dihydropyrimidin-2(1H)-one (2)

Yield: $65 \%$; M.p.: $220-222{ }^{\circ} \mathrm{C}$; IR $(\mathrm{KBr}) \mathrm{cm}^{-1}: 3337$ (NH str.), $1700(\mathrm{C}=\mathrm{O}), 1654(\mathrm{C}=\mathrm{O}), 1618(\mathrm{C}=\mathrm{C}), 1126$ $(\mathrm{C}-\mathrm{O}) ;{ }^{1} \mathrm{H}$ NMR $\left(500 \mathrm{MHz}, \mathrm{DMSO}-d_{6}\right): \delta=3.83(15 \mathrm{H}$, s, $\left.5 \times-\mathrm{OCH}_{3}\right), 5.68(1 \mathrm{H}, \mathrm{d}, J=2.0 \mathrm{~Hz}, \mathrm{H}-4) ; 6.73-7.55$ $(5 \mathrm{H}, \mathrm{m}, \mathrm{Ar}-\mathrm{H}), 8.31(1 \mathrm{H}, \mathrm{s},=\mathrm{CH}), 9.18(1 \mathrm{H}, \mathrm{bs}, \mathrm{NH}$, $\mathrm{D}_{2} \mathrm{O}$ exchg.), 10.20 (1H, bs, NH, $\mathrm{D}_{2} \mathrm{O}$ exchg.); ${ }^{13} \mathrm{C}$ NMR $\left(125.76 \mathrm{MHz}, \mathrm{DMSO}-d_{6}\right): \delta=49.5,56.1,56.5,56.7$, $60.5,60.6,60.7,99.9,106.2,112.0,112.6,120.2,124.3$, 137.5, 140.3, 142.2, 146.6, 151.4, 152.9, 153.0, 190.9; MS: $\mathrm{m} / \mathrm{z}=428.6[\mathrm{M}]+$; Analysis: for $\mathrm{C}_{22} \mathrm{H}_{24} \mathrm{~N}_{2} \mathrm{O}_{7}$, calcd. $\mathrm{C}$ 61.67, H 5.65, N 6.54\%; found C 61.86, H 5.64, N 6.55\%.

4-(2,4-Dimethoxyphenyl-5-(3,4,5-trimethoxybenzoyl)-3,4dihydropyrimidin-2(1H)-one (3)

Yield: $68 \%$; M.p.:215-217 ${ }^{\circ} \mathrm{C}$; IR (KBr) $\mathrm{cm}^{-1}: 3411$ (NH str.), $1700(\mathrm{C}=\mathrm{O}), 1654(\mathrm{C}=\mathrm{O}), 1610(\mathrm{C}=\mathrm{C}), 1126$ $(\mathrm{C}-\mathrm{O}) ;{ }^{1} \mathrm{H}$ NMR $\left(500 \mathrm{MHz}, \mathrm{DMSO}-\mathrm{d}_{6}\right): \delta=3.75(15 \mathrm{H}$, s, $\left.5 \times-\mathrm{OCH}_{3}\right), 5.58(1 \mathrm{H}, \mathrm{d}, J=2.0 \mathrm{~Hz}, \mathrm{H}-4) ; 6.24-7.17$ $(5 \mathrm{H}, \mathrm{m}, \mathrm{Ar}-\mathrm{H}), 8.31(1 \mathrm{H}, \mathrm{s},=\mathrm{CH}), 9.14(1 \mathrm{H}, \mathrm{bs}, \mathrm{NH}$, $\mathrm{D}_{2} \mathrm{O}$ exchg.), 10.20 (1H, bs, NH, $\mathrm{D}_{2} \mathrm{O}$ exchg.); ${ }^{13} \mathrm{C} \mathrm{NMR}$ $\left(125.76 \mathrm{MHz}, \mathrm{DMSO}-d_{6}\right): \delta=55.6,55.9,56.7,60.5,60.7$, 104.9, 106.2, 108.2, 142.7, 153.0, 153.2, 153.3, 190.0; MS: $\mathrm{m} / \mathrm{z}=428.5[\mathrm{M}]+;$ Analysis: for $\mathrm{C}_{22} \mathrm{H}_{24} \mathrm{~N}_{2} \mathrm{O}_{7}$, calcd. C 61.67, H 5.65, N 6.54\%; found C 61.43, H 5.66, N 6.55\%.

4-(2,4,5-Trimethoxyphenyl-5-(3,4,5-trimethoxybenzoyl)-3,4-dihydropyrimidin-2(1H)-one (4)

Yield: 70\%; M.p.: $230-232{ }^{\circ} \mathrm{C}$; IR $(\mathrm{KBr}) \mathrm{cm}^{-1}: 3412$ (NH str.), $1700(\mathrm{C}=\mathrm{O}), 1654(\mathrm{C}=\mathrm{O}), 1617(\mathrm{C}=\mathrm{C}), 1125$ $(\mathrm{C}-\mathrm{O}) ;{ }^{1} \mathrm{H}$ NMR $\left(500 \mathrm{MHz}, \mathrm{DMSO}-d_{6}\right): \delta=3.76(18 \mathrm{H}$, s, $\left.6 \times-\mathrm{OCH}_{3}\right), 5.59(1 \mathrm{H}, \mathrm{d}, J=2.0 \mathrm{~Hz}, \mathrm{H}-4) ; 6.71-7.33$ $(4 \mathrm{H}, \mathrm{m}, \mathrm{Ar}-\mathrm{H}), 8.30(1 \mathrm{H}, \mathrm{s},=\mathrm{CH}), 9.14(1 \mathrm{H}, \mathrm{bs}, \mathrm{NH}$, $\mathrm{D}_{2} \mathrm{O}$ exchg.), 10.20 (1H, bs, NH, $\mathrm{D}_{2} \mathrm{O}$ exchg.); ${ }^{13} \mathrm{C}$ NMR $\left(125.76 \mathrm{MHz}, \mathrm{DMSO}-d_{6}\right): \delta=56.4,56.7,60.7,108.2$, 131.6, 153.3, 190.0; MS: $\mathrm{m} / \mathrm{z}=458.7$ [M]+; Analysis: for $\mathrm{C}_{23} \mathrm{H}_{26} \mathrm{~N}_{2} \mathrm{O}_{8}$, calcd. C 60.26, H 5.72, $\mathrm{N}$ 6.11\%; found C $60.40, \mathrm{H} 5.73, \mathrm{~N} 6.10 \%$.

4-(2, 4,6-Trimethoxyphenyl-5-(3,4,5-trimethoxybenzoyl)-3,4-dihydropyrimidin-2(1H)-one (5)

Yield: $70 \%$; M.p.:240-242 ${ }^{\circ} \mathrm{C}$; IR $(\mathrm{KBr}) \mathrm{cm}^{-1}: 3412$ (NH str.), $1700(\mathrm{C}=\mathrm{O}), 1654(\mathrm{C}=\mathrm{O}), 1617(\mathrm{C}=\mathrm{C})$, $1125(\mathrm{C}-\mathrm{O}) ;{ }^{1} \mathrm{H}$ NMR $\left(500 \mathrm{MHz}, \mathrm{DMSO}-d_{6}\right): \delta=$ $3.78\left(18 \mathrm{H}, \mathrm{s}, 6 \times-\mathrm{OCH}_{3}\right), 6.09(1 \mathrm{H}, \mathrm{d}, J=2.0 \mathrm{~Hz}, \mathrm{H}-4)$; 6.67-7.14 $(4 \mathrm{H}, \mathrm{m}, \mathrm{Ar}-\mathrm{H}), 8.30(1 \mathrm{H}, \mathrm{s},=\mathrm{CH}), 9.14(1 \mathrm{H}$, bs, $\mathrm{NH}, \mathrm{D}_{2} \mathrm{O}$ exchg.), 10.20 (1H, bs, $\mathrm{NH}, \mathrm{D}_{2} \mathrm{O}$ exchg.); ${ }^{13} \mathrm{C}$ NMR $\left(125.76 \mathrm{MHz}, \mathrm{DMSO}-d_{6}\right): \delta=55.0,56.0$, 56.7, 60.7, 93.2, 108.2, 131.8, 133.8, 138.2, 131.8, 133.8,
138.2, 153.2, 153.3, 161.6, 193.1, 193.8; MS: $\mathrm{m} / \mathrm{z}=458.3$ $[\mathrm{M}]^{+}$; Analysis: for $\mathrm{C}_{23} \mathrm{H}_{26} \mathrm{~N}_{2} \mathrm{O}_{8}$, calcd. C 60.26, H 5.72, N 6.11\%; found C 60.45, H 5.71, N 6.12\%.

4-(3,4,5-Trimethoxyphenyl-5-(3,4,5-trimethoxybenzoyl)-3,4-dihydropyrimidin-2(1H)-one (6)

Yield: 72\%; M.p.:218-220 ${ }^{\circ} \mathrm{C}$; IR (KBr) cm $\mathrm{cm}^{-1}: 3412$ (NH str.), $1700(\mathrm{C}=\mathrm{O}), 1654(\mathrm{C}=\mathrm{O}), 1618(\mathrm{C}=\mathrm{C}), 1126$ $(\mathrm{C}-\mathrm{O}) ;{ }^{1} \mathrm{H}$ NMR $\left(500 \mathrm{MHz}, \mathrm{DMSO}-d_{6}\right): \delta=3.79(18 \mathrm{H}$, s, $\left.6 \times-\mathrm{OCH}_{3}\right), 5.38(1 \mathrm{H}, \mathrm{d}, J=2.5 \mathrm{~Hz}, \mathrm{H}-4) ; 6.64-7.81$ $(4 \mathrm{H}, \mathrm{m}, \mathrm{Ar}-\mathrm{H}), 8.30(1 \mathrm{H}, \mathrm{s},=\mathrm{CH}), 9.10(1 \mathrm{H}, \mathrm{bs}, \mathrm{NH}$, $\mathrm{D}_{2} \mathrm{O}$ exchg.), $9.90\left(1 \mathrm{H}, \mathrm{bs}, \mathrm{NH}, \mathrm{D}_{2} \mathrm{O}\right.$ exchg.); ${ }^{13} \mathrm{C}$ NMR $\left(125.76 \mathrm{MHz}, \mathrm{DMSO}-d_{6}\right): \delta=56.2,56.5,60.5,104.2$, 106.1, 134.4, 140.3, 153.0, 153.3; MS: $\mathrm{m} / \mathrm{z}=458.2[\mathrm{M}]^{+}$; Analysis: for $\mathrm{C}_{23} \mathrm{H}_{26} \mathrm{~N}_{2} \mathrm{O}_{8}$, calcd. C $60.26, \mathrm{H} 5.72, \mathrm{~N}$ 6.11\%; found $\mathrm{C} 60.46, \mathrm{H} 5.71, \mathrm{~N} 6.10 \%$.

4-(3,4-Diethoxyphenyl-5-(3,4,5-trimethoxybenzoyl)-3,4dihydropyrimidin-2(1H)-one (7)

Yield: 60\%; M.p.:190-192 ${ }^{\circ} \mathrm{C}$; IR (KBr) cm ${ }^{-1}: 3412$ (NH str.), $1700(\mathrm{C}=\mathrm{O}), 1647(\mathrm{C}=\mathrm{O}), 1617(\mathrm{C}=\mathrm{C})$, $1125(\mathrm{C}-\mathrm{O}) ;{ }^{1} \mathrm{H}$ NMR $\left(500 \mathrm{MHz}, \mathrm{DMSO}-d_{6}\right): \delta=1.30$ $\left(6 \mathrm{H}, \mathrm{s}, 2 \times-\mathrm{CH}_{3}\right), 3.89\left(9 \mathrm{H}, \mathrm{s}, 3 \times-\mathrm{OCH}_{3}\right), 4.00(4 \mathrm{H}, \mathrm{s}$, $\left.2-\mathrm{OCH}_{2}\right), 5.35(1 \mathrm{H}, \mathrm{d}, J=2.5 \mathrm{~Hz}, \mathrm{H}-4) ; 6.74-7.52(5 \mathrm{H}$, $\mathrm{m}, \operatorname{Ar}-\mathrm{H}), 7.80(1 \mathrm{H}, \mathrm{s},=\mathrm{CH}), 9.23\left(1 \mathrm{H}, \mathrm{bs}, \mathrm{NH}, \mathrm{D}_{2} \mathrm{O}\right.$ exchg.), 9.82 (1H, bs, $\mathrm{NH}, \mathrm{D}_{2} \mathrm{O}$ exchg.); $\mathrm{MS}: \mathrm{m} / \mathrm{z}=456.6$ $[\mathrm{M}]^{+}$; Analysis: for $\mathrm{C}_{24} \mathrm{H}_{28} \mathrm{~N}_{2} \mathrm{O}_{7}$, calcd. C 63.15, H 6.18, $\mathrm{N} 6.14 \%$; found C 63.35, H 6.20, N 6.12\%.

4-(4-Ethoxy,3-methoxyphenyl-5-(3,4,5-trimethoxybenzoyl)-3,4-dihydropyrimidin-2(1H)-one (8)

Yield: 50\%; M.p.:198-200 ${ }^{\circ} \mathrm{C}$; IR (KBr) cm $\mathrm{cm}^{-1}: 3411$ (NH str.), $1700(\mathrm{C}=\mathrm{O}), 1654(\mathrm{C}=\mathrm{O}), 1617(\mathrm{C}=\mathrm{C}), 1126$ $(\mathrm{C}-\mathrm{O}) ;{ }^{1} \mathrm{H}$ NMR $\left(500 \mathrm{MHz}, \mathrm{DMSO}-d_{6}\right): \delta=1.37(3 \mathrm{H}$, $\left.\mathrm{s},-\mathrm{CH}_{3}\right), 3.84\left(12 \mathrm{H}, \mathrm{s}, 4 \times-\mathrm{OCH}_{3}\right), 4.00\left(2 \mathrm{H}, \mathrm{s},-\mathrm{OCH}_{2}\right)$, $5.34(1 \mathrm{H}, \mathrm{d}, J=2.5 \mathrm{~Hz}, \mathrm{H}-4) ; 6.74-7.80(5 \mathrm{H}, \mathrm{m}, \mathrm{Ar}-$ $\mathrm{H}), 8.31(1 \mathrm{H}, \mathrm{s},=\mathrm{CH}), 9.22\left(1 \mathrm{H}, \mathrm{bs}, \mathrm{NH}, \mathrm{D}_{2} \mathrm{O}\right.$ exchg. $)$, $9.90\left(1 \mathrm{H}, \mathrm{bs}, \mathrm{NH}, \mathrm{D}_{2} \mathrm{O}\right.$ exchg.); ${ }^{13} \mathrm{C}$ NMR $(125.76 \mathrm{MHz}$, DMSO $\left.-d_{6}\right): \delta=53.5,55.9,56.4,56.5,56.7,60.5,60.7$, 60.2, 106.1, 108.2, 111.1, 112.4, 113.7, 118.7, 134.4, 136.9, 140.3, 142.3, 142.1, 147.0, 149.2, 151.7, 152.9, 153.0, 153.2, 191.1; MS: $\mathrm{m} / \mathrm{z}=442.5[\mathrm{M}]^{+}$; Analysis: for $\mathrm{C}_{23} \mathrm{H}_{26} \mathrm{~N}_{2} \mathrm{O}_{7}$, calcd. C 62.43, H 5.92, N 6.33\%; found C 62.66, H 5.91, $\mathrm{N} 6.34 \%$.

4-(4-Hydroxy-3,5-Dimethoxyphenyl-5-(3,4,5-trimethoxybenzoyl)-3,4-dihydropyrimidin-2(1H)-one (9)

Yield: $45 \%$; M.p.: $220-222{ }^{\circ} \mathrm{C}$; IR $(\mathrm{KBr}) \mathrm{cm}^{-1}: 3420$ (NH str.), $1700(\mathrm{C}=\mathrm{O}), 1654(\mathrm{C}=\mathrm{O}), 1617(\mathrm{C}=\mathrm{C}), 1126$ $(\mathrm{C}-\mathrm{O}) ;{ }^{1} \mathrm{H}$ NMR $\left(500 \mathrm{MHz}, \mathrm{DMSO}-d_{6}\right): \delta=3.9(15 \mathrm{H}$, s, $\left.5 \times-\mathrm{OCH}_{3}\right), 5.30(1 \mathrm{H}, \mathrm{d}, J=2.0 \mathrm{~Hz}, \mathrm{H}-4) ; 6.70-7.14$ $(4 \mathrm{H}, \mathrm{m}, \mathrm{Ar}-\mathrm{H}), 8.30(1 \mathrm{H}, \mathrm{s},=\mathrm{CH}), 9.20(1 \mathrm{H}, \mathrm{bs}, \mathrm{NH}$, $\mathrm{D}_{2} \mathrm{O}$ exchg.), $10.00\left(1 \mathrm{H}\right.$, bs, $\mathrm{NH}, \mathrm{D}_{2} \mathrm{O}$ exchg.); ${ }^{13} \mathrm{C}$ NMR $\left(125.76 \mathrm{MHz}, \mathrm{DMSO}-d_{6}\right): \delta=56.5,56.6,60.7,108.2$, 131.8, 133.8, 138.2, 142.5, 153.2, 193.8; MS: $\mathrm{m} / \mathrm{z}=444.7$ $[\mathrm{M}]^{+}$; Analysis: for $\mathrm{C}_{22} \mathrm{H}_{24} \mathrm{~N}_{2} \mathrm{O}_{8}$, calcd. C 59.45, H 5.44, $\mathrm{N} 6.30 \%$; found C 59.67, H 5.45, N 6.32\%.

\section{Biological Evaluation}

HepG2 hepatocellular carcinoma cells were maintained in RPMI 1640 (Sigma). The HepG2 cells were grown in 96-well plates. Vibrant apoptosis assay kit (Annexin-V, APC conjugate; Molecular Probes ${ }^{\mathrm{TM}}$ ) was applied to study cell viability as per the manufacturer's recommendation. Briefly, the study was performed on both adherent and floating cells after collection. 

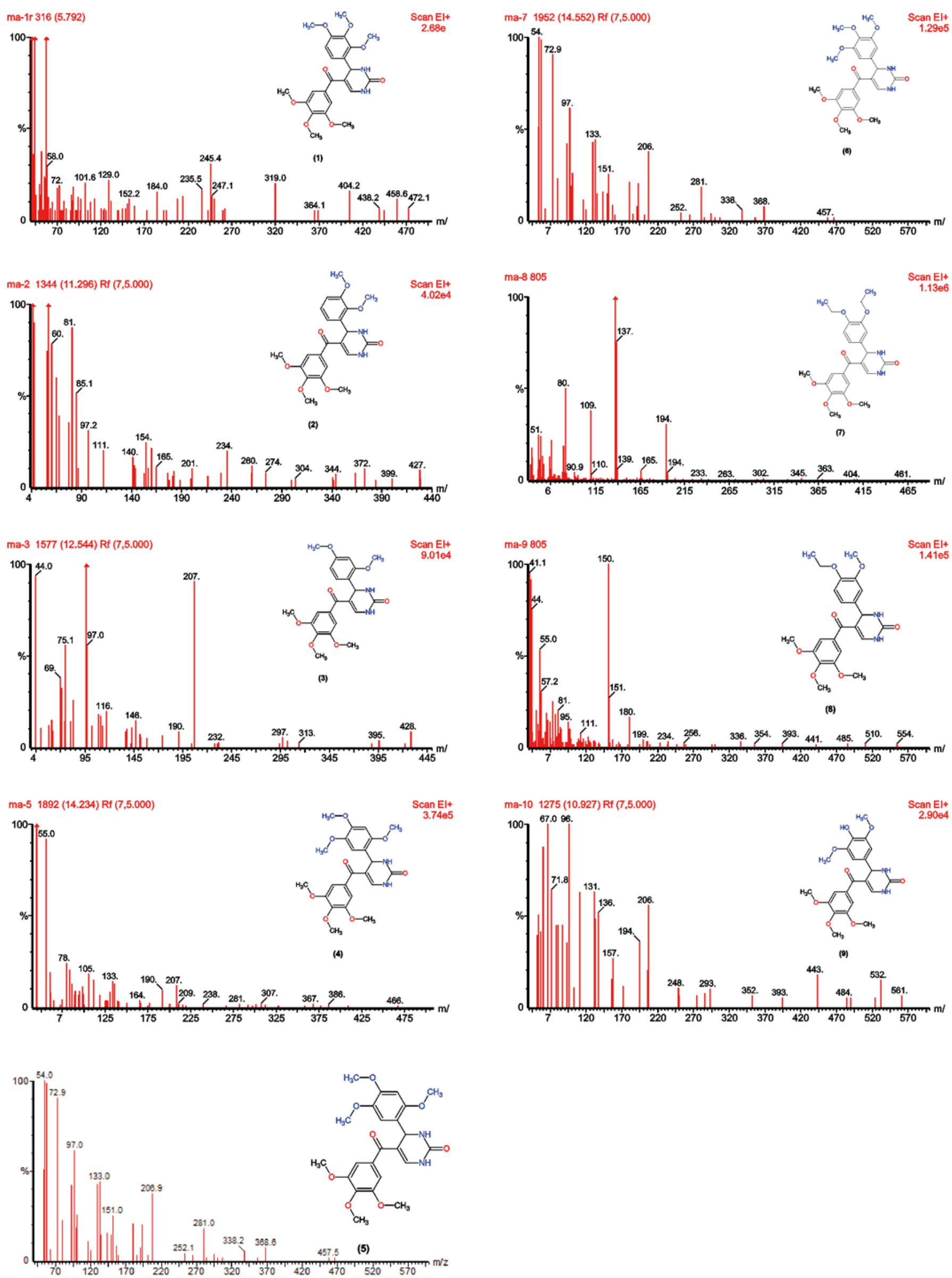

Figure 1. Mass spectra of compounds (1-9) 
DAPI (4',6-diamidino-2-phenylindole) was used as a viability dye. Fluorescence was analyzed on a total of $10^{4}$ cells per sample using a flow cytometer and cells were considered viable if they were double negative for Annexin-V and DAPI.

\section{Flow Cytometric analysis of cellular DNA content:}

HepG2 cells were fixed in $1 \mathrm{~mL}$ ethanol (70\%) for 1 hour at $25{ }^{\circ} \mathrm{C}$. $1 \mathrm{~mL}$ Sodium citrate $(50 \mathrm{mM})$ containing $250 \mu \mathrm{g}$ RNase was used for harvested HepG2 for harvesting and incubated for 1 hour at $50{ }^{\circ} \mathrm{C}$. Further, propidium iodine (PI) in the same buffer was used and cells were incubated for half-hour. Finally, the HepG2 cells were analyzed by flow cytometry (Becton Dickinson, San Jose, CA, USA).

\section{RESULTS AND DISCUSSION}

Enaminone, (2E)-3-(dimethylamino)-1-(3,4,5-trimethoxyphenyl) prop-2-en-1-one (II) was synthesized by refluxing 3,4,5-trimethoxy acetophenone (I) with dimethylforamide dimethylacetal (DMF-DMA) without solvent for $12 \mathrm{~h}$ (Scheme 1). Dihydropyrimidinone derivatives (1-9) were synthesized by refluxing enaminone (II) $(0.01 \mathrm{~mol})$, substituted benzaldehyde $(0.01 \mathrm{~mol})$, urea $(0.01 \mathrm{~mol})$ and glacial acetic acid $(10 \mathrm{~mL})$ for $3 \mathrm{~h}$. The synthesized compounds were characterized and confirmed by spectroscopical methods. Two singlets at $\delta \mathrm{H} 2.93,3.14 \mathrm{ppm}$ due to the $N$, $N$-dimethyl protons and two doublets at $\delta \mathrm{H} 5.83$ and 7.71 $\operatorname{ppm}(\mathrm{d}, J=12 \mathrm{~Hz})$ due to the ethylenic protons were ob-

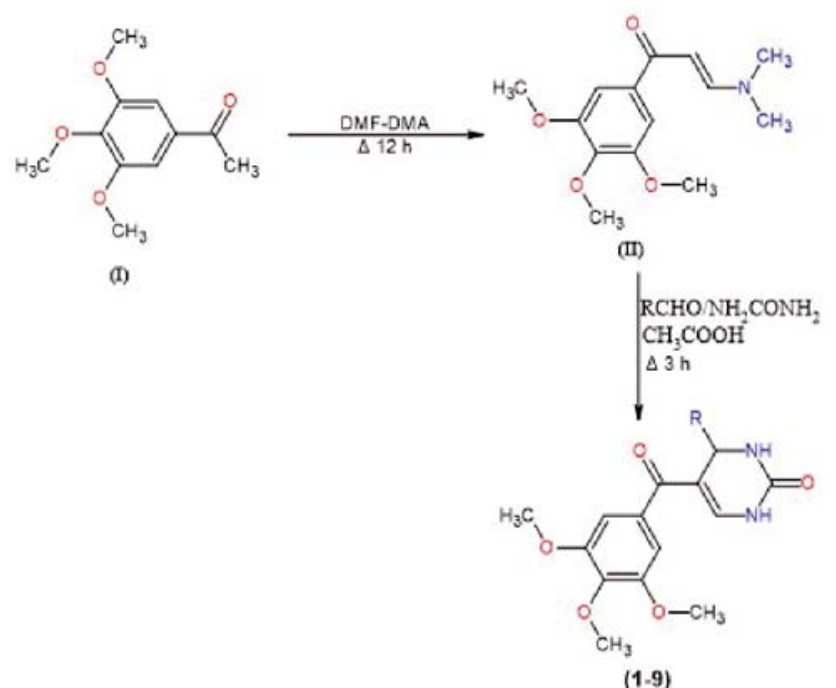

Compounds

$\mathrm{R}$

\section{1}

2

3

4

5

6

7

8

9
2,3,4-Trimethoxyphenyl

2,3-Dimethoxyphenyl

2,4-Dimethoxypheny1

2,4,5-Trimethoxyphenyl

2,4,6-Trimethoxyphenyl

3,4,5-Trimethoxyphenyl

3,4-Diethoxyphenyl

3-Methoxy-4-ethoxyphenyl

4-Hydroxy-3,5-dimethoxyphenyl

Scheme 1. Reaction scheme for the synthesis of dihydropyrimidinone derivatives (1-9) served in ${ }^{1} \mathrm{H}$ NMR spectrum of enaminone. The coupling constant $(J=12 \mathrm{~Hz})$ for the ethylenic protons confirmed that the enaminone existed in the $E$-configuration ${ }^{24}$. Two $\mathrm{NH}$ protons of dihydropyrimidinones were observed as exchangeable protons at $\delta$ 9.1-9.2 ppm and $\delta$ 9.8-10.2 ppm. The H-4 protons of dihydropyrimidinone were observed at $\delta$ 5.3-5.6 $\mathrm{ppm}^{25}$. In GC/MS analysis, all the synthesized compounds present molecular ion peaks according to their molecular weights (Fig. 1). The analysis of compounds by spectroscopic techniques confirmed the synthesized compounds (1-9). The possible reaction mechanism of dihydropyrimidinone synthesis involves the acid-catalyzed formation of iminium ion intermediate (Scheme 2).

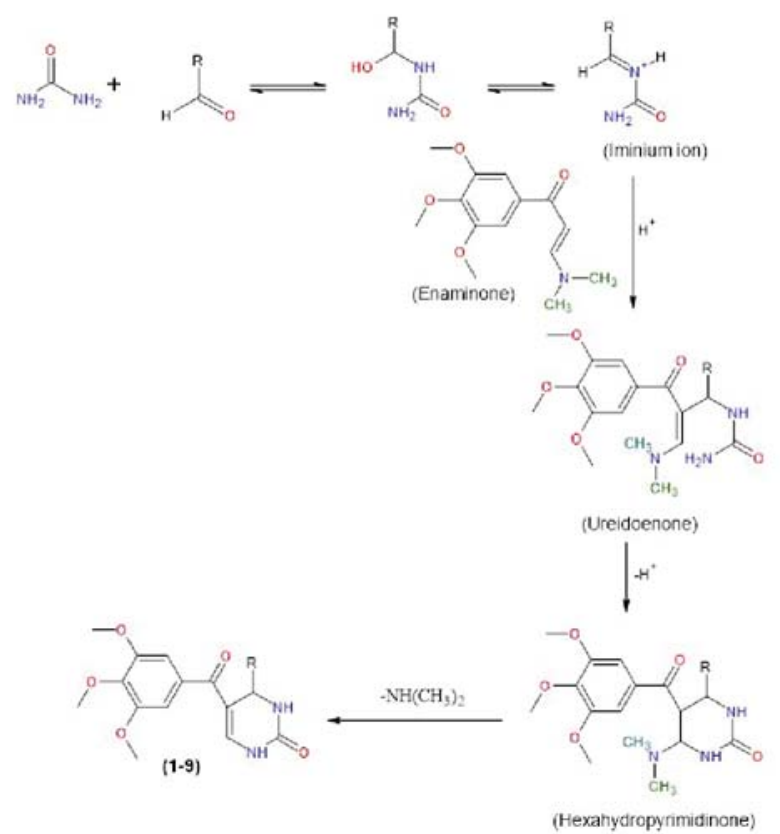

Scheme 2. The possible mechanism for the synthesis of dihydropyrimidinone derivatives containing 3,4,5-timethoxybenzoyl moiety

In vitro cytotoxicity evaluation

All compounds of this series were evaluated as cytotoxic to HepG2 hepatocellular carcinoma cell line using $10 \mu \mathrm{M}$ concertation. Trypan blue Cell stain was used to assess cell viability using the dye exclusion test. However only compound $\mathbf{9}$ was active to induce cell death and other compounds showed no activity on cell viability or cell proliferation (Fig. 2, Fig. 3). Therefore, compound 9 was selected for further biological evaluation.

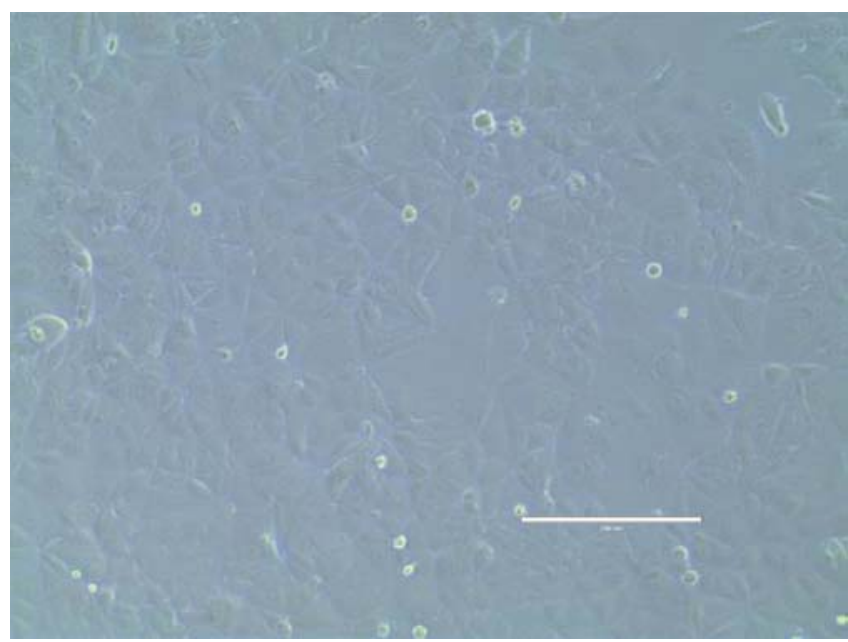

Figure 2. Photograph showing untreated HepG2 cancer cells 


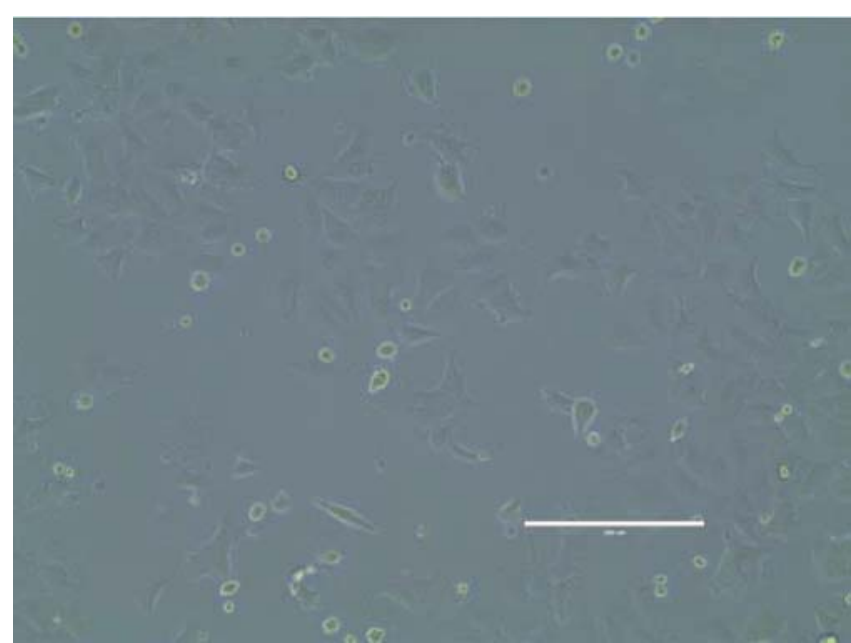

Figure 3. Photograph showing treated HepG2 cancer cells

\section{Apoptosis assay}

A hallmark of apoptosis is the exposure of phosphatidylserine on the surface of apoptotic cells, which mediates their recognition and phagocytosis by macrophages. Caspases, a family of cysteine proteases, are specifically activated in apoptosis and mediate the series of characteristic morphological changes. Extensive research has proved that the features of apoptotic cells may vary significantly depending on the cell type, the nature of

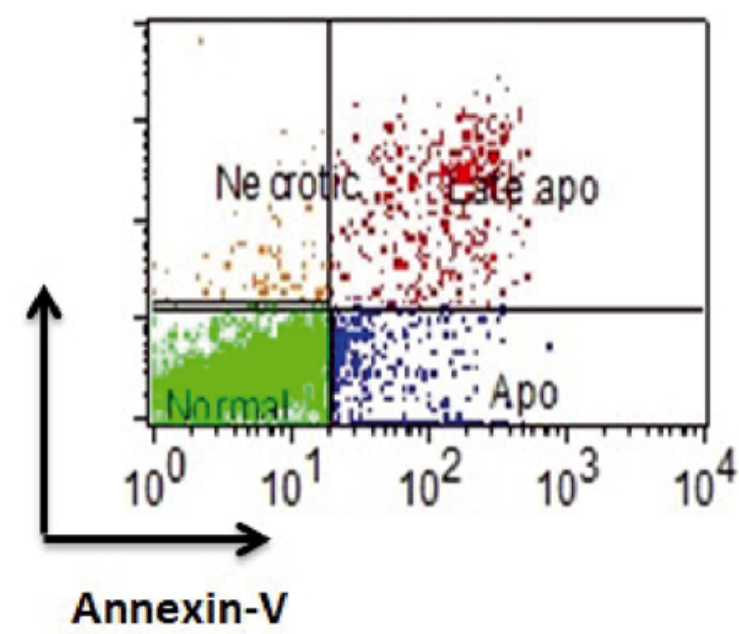

the apoptotic inducers and the stage of apoptosis to be tested.

In the present study, the effect of compound 9 was investigated using HepG2 cancer cells. After Annexin V and DAPI staining, cells were analyzed by flow cytometry (Fig. 4). The results revealed that the treatment did not increase the number of apoptotic cells compared to the control. However, it showed a significant increase in necrosis $\%$ from $1.97 \%$ to $12.18 \%$.

\section{Cell cycle distribution}

Fluorescence-activated cell sorting (FACS) analysis was used to study the effect of drug treatment on cell cycle distribution. HepG2 cells were treated with compound $9(10 \mu \mathrm{M})$ for $48 \mathrm{~h}$. The analysis showed no dramatic change in the accumulation of $\mathrm{G} 1$ and $\mathrm{S}$ phases. However, there is a decrease in $\mathrm{G} 2+\mathrm{M}$ from 12.90 to 8.13 (Fig. 5).

\section{CONCLUSION}

In conclusion, novel dihydropyrimidinone derivatives (1-9) were synthesized in good yields. The enaminone (II) used as a starting material was obtained without solvent by reaction of 3,4,5-trimethoxy acetophenone (I) with dimethylforamide dimethylacetal (DMF-DMA). The final novel dihydropyrimidinones $(1-9)$ were obtained by reacting the enaminone with substituted benzaldehydes,

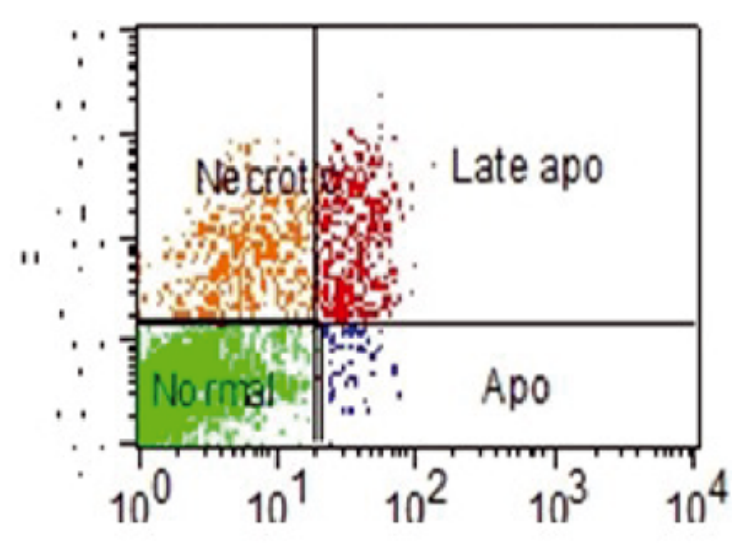

Figure 4. HepG2 cells were treated and analyzed at 0 h, and 24 h by Annexin-V/PI apoptosis kit flow cytometry. Histograms show the PI positive cells per channel (vertical axis) vs. Annexin-V (horizontal axis) positive cells
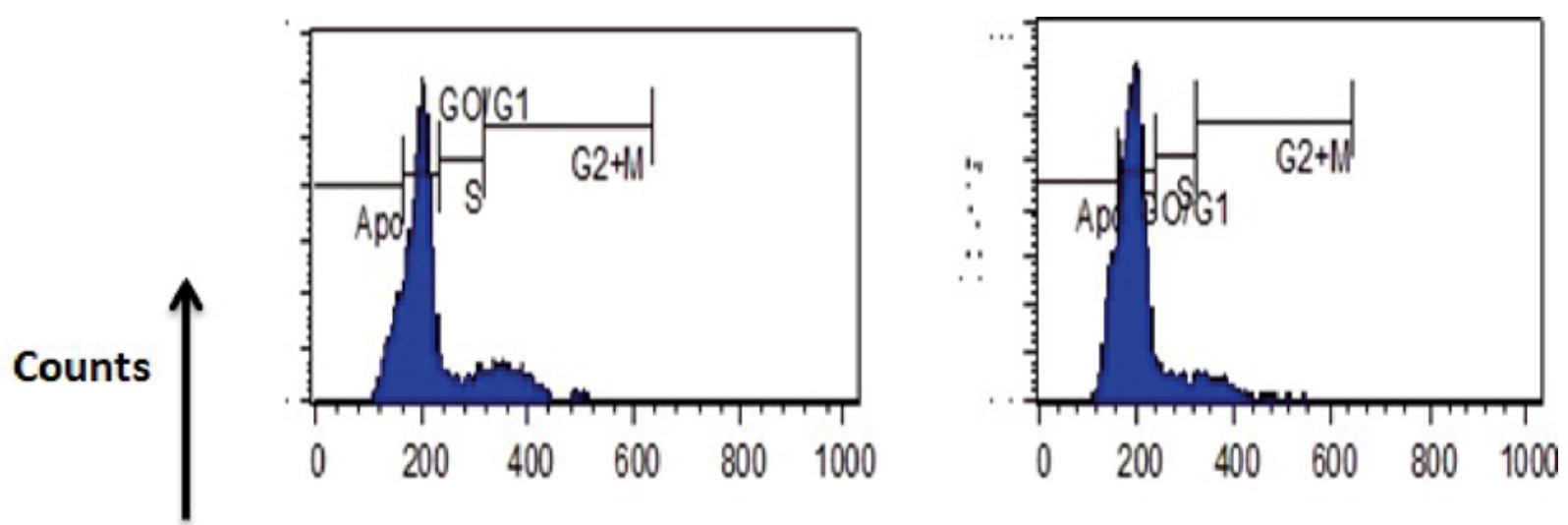

PI

Figure 5. HepG2 cells were treated and analyzed at $0 \mathrm{~h}$, and $24 \mathrm{~h}$ by DNA cycle analysis flow cytometry. Histograms show the number of cells per channel (vertical axis) vs. DNA content (horizontal axis) 
urea and glacial acetic acid. All the prepared compounds were analyzed and confirmed by several spectroscopic techniques. All the compounds were screened for anticancer activity against HepG2 cancer cell line. Only compound 9 displayed significant anti-cancer activity. During apoptotic assay, it showed a significant increase in necrosis from $1.97 \%$ to $12.18 \%$ as compared to control. Mechanism of anti-proliferation by cell cycle distribution assay also confirmed that there is a decrease in $\mathrm{G} 2+\mathrm{M}$ from 12.90 to 8.13 as compared to control.

\section{ACKNOWLEDGMENTS}

Authors are grateful to King Saud University, Riyadh, Saudi Arabia for funding the work through Researchers Supporting Project (No. RSP-2021/359).

\section{LITERATURE CITED}

1. Folkers, K., Harwood, H.J. \& Johnson, T.B. (1932). Researches on pyrimidines. cxxx. synthesis of 2-keto-1,2,3,4tetrahydropyrimidines. J. Am. Chem. Soc. 54, 3751. DOI: 10.1021/ja01348a040.

2. Atwal, K.S., Ahmed, S.Z., Bird, J.E., Delaney, C.L., Dickinson, K.E., Ferrara, F.N., Hedberg, A., Miller, A.V., Moreland, S. \& O'Reilly, B.C. (1992). Dihydropyrimidine angiotensin II receptor antagonists. J. Med. Chem. 35, 4751-4763. DOI: 10.1021/jm00103a014.

3. Rana, K., Kaur, B. \& Kumar, B. (2004). Synthesis and antihypertensive activity of some dihydropyrimidines. Indian J. Chem. 43, 1553-1557.

4. Rovnyak, G.C., Kimball, S.D., Beyer, B., Cucinotta, G., DiMarco, J.D., Gougoutas, J., Hedberg, A., Malley, M., McCarthy, J.P., Zhang, R. \& Moreland, S. (1995). Calcium entry blockers and activators: conformational and structural determinants of dihydropyrimidine calcium channel modulators. $J$. Med. Chem. 38, 119-29. DOI: 10.1021/jm00001a017.

5. Atwal, K.S., Swanson, B.N., Unger, S.E., Floyd, D.M., Moreland, S., Hedberg, A. \& Reilly, B.C.O. (1991). Dihydropyrimidine calcium channel blockers. 3. 3-Carbamoyl-4-aryl1,2,3,4-tetrahydro-6-methyl-5-pyrimidinecarboxylic acid esters as orally effective antihypertensive agents. J. Med. Chem. 34, 806-811. DOI: 10.1021/jm00106a048.

6. Kappe, C.O. (1993). 100 years of the Biginelli dihydropyrimidine synthesis. Tetrahedron, 49, 6937-6963. DOI: 10.1016/ S0040-4020(01)87971-0.

7. Beena, K.P., Suresh, R., Rajasekaran, A. \& Manna, P.K. (2016). Dihydropyrimidinones- a versatile Scaffold with diverse biological activity. J. Pharm. Sci. \& Res. 8, 741-746.

8. Jalali, M., Mahdavi, M., Memarian, H.R., Ranjbar, M., Soleymani, M., Fassihi, A. \& Abedi, D. (2012). Antimicrobial evaluation of some novel derivatives of 3,4-dihydropyrimidine2(1H)-One. Res. Pharm. Sci. 7, 243-247. PMID: 23248675.

9. Pramanik, T., Pathan, A.H., Gupta, R., Singh, J. \& Singh, S. (2015). Dihydropyrimidinone derivatives: green synthesis and effect of electronic factor on their antimicrobial properties. Res. J. Pharm. Biol. Chem. Sci. 6, 1152-1157.

10. Shaikh, A. \& Meshram, J.S. (2015). Design, synthesis and pharmacological assay of novel azo derivatives of dihydropyrimidinones. Cogent Chem. 1, 1019809. DOI:10.1080/23 312009.2015.1019809.

11. Liu, Y., Liu, J., Zhang, R., Guo, Y., Wang, H., Meng, Q., Sun, Y. \& Liu, Z. (2019). Synthesis, characterization, and anticancer activities evaluation of compounds derived from 3,4-dihydropyrimidin-2(1H)-One. Molecules, 24, 891. DOI: 10.3390/molecules24050891.

12. Abdel-Latif, N.A., Sabry, N.M., Mohamed, A.M. \& Abdulla, M.M. (2007). Synthesis, analgesic, and antiparkinsonian profiles of some pyridine, pyrazoline, and thiopyrimidine de- rivatives. Monatshefte fur Chemie., 138, 715-724. DOI: 10.1007/ s00706-007-0656-8.

13. Bairagi, K.M., Younis, N.S., Emeka, P.M., Sangtani, E., Gonnade, R.G., Venugopala, K.N., Alwassil, O.I., Khalil, H.E. \& Nayak, S.K. (2020). Antidiabetic activity of dihydropyrimidine scaffolds and structural insight by single crystal x-ray studies. Med. Chem. 16, 996-1003. DOI: 10.2174/15734064166661912 27123048.

14. Farghaly, A.M., AboulWafa, O.M., Elshaier, Y.A.M., Badawi, W.A., Haridy, H.H. \& Mubarak, H.A.E. (2019). Design, synthesis, and antihypertensive activity of new pyrimidine derivatives endowing new pharmacophores. Med. Chem. Res. 28, 360-379. DOI: 10.1007/s00044-019-02289-6.

15. Silva, G.C.O., Correa, J.R., Rodrigues, M.O., Alvim, H.G.O., Guido, B.C., Gatto, C.C., Wanderley, K.A., Fioramonte, M., Gozzo, F.C., De Souza, R.O.M.A. \& Neto, B.A.D. (2015). The Biginelli reaction under batch and continuous flow conditions: catalysis, mechanism and antitumoral activity. RSC $A d v$. 5, 48506-48515. DOI: 10.1039/c5ra07677c.

16. Soumyanarayanan, U., Bhat, V.G., Kar, S.S. \& Mathew J.A. (2012). Monastrol mimic Biginelli dihydropyrimidinone derivatives: synthesis, cytotoxicity screening against HepG2 and HeLa cell lines and molecular modeling study. Org. Med. Chem. Lett., 2, 23. DOI: 10.1186/2191-2858-2-23.

17. Romagnoli, R., Baraldi, P.G., Cara, C.L., Hamel, E., Basso, G., Bortolozzi, R. \& Viola, G. (2010). Synthesis and biological evaluation of 2-(3',4',5'-trimethoxybenzoyl)-3-aryl/ arylaminobenzo[b]thiophene derivatives as a novel class of antiproliferative agents. Eur. J. Med. Chem. 45, 5781-5791. DOI: 10.1016/j.ejmech.2010.09.038.

18. Tseng, H.H., Chuah, Q.Y., Yang, P.M., Chen, C.T., Chao, J.C., Lin, M.D. \& Chiu, S.J. (2012). Securin enhances the anticancer effects of 6-methoxy-3-(3',4',5'-trimethoxy-benzoyl)-1Hindole (BPR0L075) in human colorectal cancer cells. PLoS One, 7, e36006. DOI: 10.1371/journal.pone.0036006.

19. Bhat, M.A., Al-Omar, M.A., Ghabbour, H. \& Naglah, A. (2018). A one-pot Biginelli synthesis and sharacterization of novel dihydropyrimidinone derivatives containing piperazine/morpholine moiety. Molecules, 23, 1559. DOI: 10.3390/ molecules23071559.

20. Bhat, M. A., Al-Omar, M. A., Naglah., A. \& Al-Dhfyan, A. (2020). Biginelli synthesis of novel dihydropyrimidinone derivatives containing phthalimide moiety. J. Chem. 2020, 1-10. DOI: $10.1155 / 2020 / 4284628$.

21. Bhat, M.A., Al-Omar, M.A., Naglah, A. \& Khan, A.A. (2019). Enaminone-derived pyrazoles with antimicrobial activity. J. Chem. 2019, 1-10. DOI: 10.1155/2019/2467970.

22. Bhat, M.A., Al-Omar, M.A., Khan, A.A., Alanazi, A.M. \& Naglah, A.M. (2019). Synthesis and antihepatotoxic activity of dihydropyrimidinone derivatives linked with 1, 4-benzodioxane. Drug Des. Devel. Ther. 13, 2393. DOI 10.2147/DDDT.S198865.

23. Bhat, M.A., Al-Omar, M.A. \& Naglah, A. (2018). Synthesis and in vivo anti-ulcer evaluation of some novel piperidine linked dihydropyrimidinone derivatives. J. Enzyme. Inhib. Med. Chem. 33, 978-988. DOI: 10.1080/14756366.2018.1474212.

24. Bhat, M.A., Ahmed, A.F., Wen, Z.H., Al-Omar, M.A. \& Abdel-Aziz, H.A. (2017). Synthesis, anti-inflammatory and neuroprotective activity of pyrazole and pyrazolo[3,4-d] pyridazine bearing 3,4,5-trimethoxyphenyl. Med. Chem. Res. 26, 1557-1566. DOI: 10.1007/s00044-017-1870-5.

25. Bhat, M.A., Al-Omar, M.A., Naglah, A., Kalmouch, A. \& Al-Dhfyan, A. (2019). Synthesis and characterization of novel Biginelli dihydropyrimidinone derivatives containing imidazole moiety. J. Chem. 2019, 1-10. DOI:10.1155/2019/3131879. 